DRAFT VERSION SEPTEMBER 1, 2021

Preprint typeset using LTEX style emulateapj v. 5/2/11

\title{
ABELL 1201: A MINOR MERGER AT SECOND CORE PASSAGE
}

\author{
Cheng-Jiun Ma ${ }^{1,2}$, Matt Owers ${ }^{3,4}$, Paul E. J. Nulsen ${ }^{1}$, Brian R. McNamara ${ }^{1,2,5}$, Stephen S. Murray ${ }^{1,6}$, Warrick J. \\ $\mathrm{COUCH}^{4}$ \\ Draft version September 1, 2021
}

\begin{abstract}
We present an analysis of the structures and dynamics of the merging cluster Abell 1201, which has two sloshing cold fronts around a cooling core, and an offset gas core approximately $500 \mathrm{kpc}$ northwest of the center. New Chandra and XMM-Newton data reveal a region of enhanced brightness east of the offset core, with breaks in surface brightness along its boundary to the north and east. This is interpreted as a tail of gas stripped from the offset core. Gas in the offset core and the tail is distinguished from other gas at the same distance from the cluster center chiefly by having higher density, hence lower entropy. In addition, the offset core shows marginally lower temperature and metallicity than the surrounding area. The metallicity in the cool core is high and there is an abrupt drop in metallicity across the southern cold front. We interpret the observed properties of the system, including the placement of the cold fronts, the offset core and its tail in terms of a simple merger scenario. The offset core is the remnant of a merging subcluster, which first passed pericenter southeast of the center of the primary cluster and is now close to its second pericenter passage, moving at $\simeq 1000 \mathrm{~km} \mathrm{~s}^{-1}$. Sloshing excited by the merger gave rise to the two cold fronts and the disposition of the cold fronts reveals that we view the merger from close to the plane of the orbit of the offset core.
\end{abstract}

Subject headings: galaxies: clusters: general; Galaxies: clusters: individual: Abell 1201

\section{INTRODUCTION}

In hierarchical structure formation models, galaxy clusters are formed by mergers of smaller systems, including other groups and clusters, taking place over the age of the universe (e.g. Springel et al. 2006). Mergers between galaxy clusters are among the most energetic events in the universe (Sarazin 2002). Although the merger rate is now decreasing with time, evidence of recent and ongoing mergers is still commonly found in clusters. Cluster mergers can induce pronounced observable features, particularly in the X-rays. High resolution X-ray observations with Chandra and XMM-Newton provide a unique means to study cluster mergers. Combined with galaxy redshifts from optical spectroscopy and mass distributions from lensing, they provide a valuable tool for studying merger dynamics and testing our understanding of cluster physics.

A notable discovery from X-ray astrophysics in the past decade is the phenomenon of cold fronts in clusters (Markevitch et al. 2000, Vikhlinin et al. 2001). These are contact discontinuities where there is an abrupt change in the entropy of the gas (Markevitch \& Vikhlinin 2007). Simulations (e.g. Churazov et al. 2003; Tittley \& Henriksen 2005; Ascasibar \& Markevitch 2006; Poole et al.2006, 2008; ZuHone et al.2010; Roediger et al. 2011) show that cold fronts can be induced in more than one way during cluster mergers. Cold fronts of the "remnant core type" occur at the interface between the

\footnotetext{
${ }^{1}$ Harvard-Smithsonian Center for Astrophysics, 60 Garden St., Cambridge, Massachusetts, 02138-1516, USA

2 Department of Physics \& Astronomy, University of Waterloo, 200

University Ave. W., Waterloo, Ontario, N2L 3G1, Canada

${ }^{3}$ Australian Astronomical Observatory, PO Box 296, Epping, NSW 1710, Australia

${ }^{4}$ Centre for Astrophysics \& Supercomputing, Swinburne University of Technology, PO Box 218, Hawthorn, VIC 3122, Australia

${ }^{5}$ Perimeter Institute for Theoretical Physics, 31 Caroline St. N., Waterloo, Ontario, N2L 2Y5, Canada

${ }^{6}$ Department of Physics and Astronomy, Johns Hopkins University, 3400 North Charles St., Baltimore, MD 21205, USA
}

core of an infalling subcluster and the warmer ICM of the primary cluster (e.g. 1E0657-56; Markevitch et al. 2002). Another type, called "sloshing" cold fronts, result from gas motions in a cluster core induced by the gravitational perturbation of an infalling subcluster (e.g. Abell 1795; Markevitch et al. 2001). Simulations (e.g Ascasibar \& Markevitch 2006, ZuHone et al. 2010) show that the "sloshing" type of cold fronts appear $\sim 0.3 \mathrm{Gyr}$ after pericentric passage of a subcluster. This cold front moves outward and a second cold front can appear on the opposite side of the center $0.6 \mathrm{Gyr}$ or longer after pericentric passage. While the cold fronts continue to move outward, additional cold fronts may appear on alternating sides of the center. Viewed from many directions, the fronts are connected in a spiral pattern. Although the time scales vary from cluster to cluster, the well-defined structure of alternating fronts and/or a spiral, are a signature of sloshing induced by mergers. These features have been shown to be useful for constraining the dynamics and history of mergers (e.g. Johnson et al. 2010, 2011).

Abell 1201 (A1201) is a typical example of a cluster with sloshing cold fronts (Owers et al. 2009b, ONC hereafter; Owers et al. 2011b). It has a redshift of 0.168 (Struble \& $\operatorname{Rood} 1999$ ) and an X-ray luminosity of $L_{\mathrm{X}}(0.1-2.4 \mathrm{keV})=$ $2.4 \times 10^{44} \mathrm{erg} \mathrm{s}^{-1}$ (Böhringer et al. 2000; Ebeling et al. 1998). ONC measured the redshifts of 321 member galaxies and found a mean redshift of $z=0.1673 \pm 0.0002$ and a velocity dispersion of $778 \pm 36 \mathrm{~km} \mathrm{~s}^{-1}$. Using $21.5 \mathrm{ksec}$ of ACIS$\mathrm{S}$ data, ONC measured a global temperature of $5.3 \pm 0.3 \mathrm{keV}$ and an abundance of $0.34 \pm 0.10$ for A1201. They also studied the spatial and redshift distributions of the galaxies in A1201 and they identified a remnant, offset core, located at the northwest end of a bright X-ray ridge that runs through the cluster center (see also Owers et al. 2009a). Edge et al. (2003) analyzed strong lensing in A1201, using the image of an arc located over the BCG, and found that the ellipticity of the mass distribution in the cluster core exceeds that of the optical isophotes of the BCG. 
In this paper, we extend the study of ONC, using deeper Chandra and XMM-Newton data to discuss the substructure and dynamics of merging in A1201. The X-ray data reduction is discussed in Section 2. The substructures of A1201, including its cold fronts, offset core, and tail are discussed in Section 3. These results are interpreted in terms of a merger scenario presented in Section 4. Section 5 is a short summary.

For this analysis, we adopt a $\Lambda \mathrm{CDM}$ cosmology with $h_{0}=$ $0.7, \Omega_{\Lambda}=0.7$, and $\Omega_{m}=0.3$. In this cosmology, $1^{\prime \prime}$ corresponds to $2.88 \mathrm{kpc}$ at a redshift of $z=0.1673$. Positions angles (PA) are measured counterclockwise from the west.

\section{DATA REDUCTION}

A1201 was observed using the European Photon Imaging Camera (EPIC) on XMM-Newton in December 2007 (ObsID 0500760101), and the Advanced CCD Imaging Spectrometer (ACIS) on Chandra in April 2008 (ACIS-I; ObsID 9616) and in November 2003 (ACIS-S; ObsID 4216). The EPIC observations were performed in full-frame mode using the medium filter, for a total exposure time of $50 \mathrm{ksec}$. The ACIS-I and ACIS-S observations were performed in VFAINT mode for total exposure times of $47 \mathrm{ksec}$ and $40 \mathrm{ksec}$, respectively.

\subsection{X-ray Imaging}

We reprocessed the EPIC data using SAS v10.0.0 with standard settings. Light curves for the $10-12 \mathrm{keV}$ band for each of the three cameras (PN, MOS1 and MOS2) were used to filter out periods when the count rate exceeded the mean by $3 \sigma$. Images were produced for each camera, using the method of Carter \& Read (2007). Blank sky and filter-wheel closed (FWC) datasets produced by the EPIC Blank Sky team 7 were used for this purpose. Particle background is first subtracted using FWC data, scaled by the counts in regions outside each detector field of view. The same procedure is applied to the blank sky datasets, in principle, leaving only the cosmic $\mathrm{X}$ ray background $(\mathrm{CXB})$. Assuming that there are no source photons in this region, counts in annuli $10-12$ arcmin from the center of each detector are then used to scale the blank sky backgrounds to the source exposures (for both, after subtraction of the hard particle background) in order to remove CXB from the source data. Allowing for some difference in the CXB spectrum between the blank sky and source exposures, this procedure is carried out in the 4 energy bands delimited by $0.5,2.125,3.750,5.375$, and $7.0 \mathrm{keV}$. The background subtracted images are then divided by vignetted exposure maps to obtain the image (left panel of Figure 1).

The ACIS-I (ObsID 9616) and ACIS-S (ObsID 4216) data sets were reprocessed using the CIAO software package (version 4.3; Fruscione et al. 2006). Following standard practice, light curves from source free regions were used to filter out periods when the count rate differed by more than $3 \sigma$ from the mean. The ACIS-S observation is badly affected by a flare, which leaves only $21.5 \mathrm{ksec}$ of useful exposure. No significant flaring is seen in the ACIS-I observation and the cleaned exposure time is $43 \mathrm{ksec}$. Background subtraction was done using the standard blank sky datasets. The exposure map was generated for emission for an optically thin plasma with a temperature of $k T=5.3 \mathrm{keV}$, the global temperature of A1201 determined by $\mathrm{ONC}$. The combined, $0.5-7 \mathrm{keV}$, background subtracted, exposure corrected image from the two data sets,

\footnotetext{
${ }^{7}$ http://xmm.vilspa.esa.es/external/xmm_sw_cal/background/blank_sky.shtml August 2010 version.
}

TABLE 1

GLOBAL PROPERTIES

\begin{tabular}{lcccc}
\hline \hline \multicolumn{1}{c}{ Data } & $\mathrm{kT}(\mathrm{keV})$ & $\mathrm{Z}$ & $\chi^{2} / \mathrm{DoF}$ & counts $^{\mathrm{a}}$ \\
\hline ACIS-I & $5.56 \pm 0.2$ & $0.31 \pm 0.1$ & $205 / 266$ & 17762 \\
ACIS-S & $5.29 \pm 0.3$ & $0.30 \pm 0.1$ & $194 / 225$ & 16499 \\
ACIS & $5.43 \pm 0.2$ & $0.32 \pm 0.07$ & $404 / 493$ & $\ldots$ \\
MOS & $5.16 \pm 0.2$ & $0.28 \pm 0.05$ & $532 / 537$ & 38353 \\
ACIS+MOS & $5.31 \pm 0.15$ & $0.29 \pm 0.039$ & $853 / 958$ & $\ldots$ \\
Owers+08 & $5.3 \pm 0.3$ & $0.34 \pm 0.10$ & & \\
\hline a Net $0.5-7 \mathrm{keV}$ counts, after background subtraction. & &
\end{tabular}

binned by a factor of 4 , is displayed in the right panel of Figure 1.

An inset image in Figure 1 shows the regions discussed in later sections of this paper. The two cold fronts discussed by ONC and Owers et al. (2009a) are marked by red arcs. The offset core to the northwest of the cluster center is outlined by a magenta ellipse. The outer edge of the tail to the east of the offset core is marked in green. The ridge connecting the cool core and the offset core is marked by two cyan lines.

\subsection{X-ray Spectral Analysis}

All X-ray spectra were extracted in the energy range 0.5 to $7.0 \mathrm{keV}$ and grouped to ensure a minimum of 20 counts per bin. PN data were not used in the spectral analysis, since the soft background cannot be convincingly removed.

Spectra and response files for the ACIS-I and ACIS-S data were assembled using the CIAO script "specextract." Regions containing X-ray point sources were identified using the CIAO "cell_detect" task with a $3 \sigma$ threshold and excluded from all extracted spectra. The ACIS-S observation was performed at the standard focal plane temperature of $-120^{\circ} \mathrm{C}$, but the focal plane was slightly warmer $\left(-118.7^{\circ} \mathrm{C}\right)$ for the ACIS-I observation. This is expected to degrade the calibration accuracy. However, our spectral fits reveal no significant discrepancy and we assume that any calibration errors are insignificant. Background spectra were created using the standard blank sky data described in Markevitch (1998). For the MOS data, spectra were extracted using the SAS "evselect" task. The point sources identified in the ACIS-I data were excluded, but using larger, $9^{\prime \prime}$ radius, apertures. Background spectra were created using a double background subtraction approach similar to that used to make the images. Briefly, the background spectrum is assumed to be the sum of two components, a hard particle component, which is determined by scaling FWC data, and a cosmic background component. The cosmic X-ray background is determined by scaling the residual blank sky spectrum, after subtraction of the particle background, to make the net spectrum in the source exposure zero in the annulus between $10^{\prime}$ and $11^{\prime}$ centered on the cluster.

To determine a global temperature and metallicity, spectra were extracted from the elliptical region defined in Figure 4 of ONC. These were fitted with an absorbed (WABS), optically thin, thermal plasma model (MEKAL; Mewe et al. 1986), using Sherpa. The absorbing column density of neutral hydrogen was fixed at $N_{\mathrm{H}}=1.61 \times 10^{20} \mathrm{~cm}^{-2}$, allowing for foreground gas (Dickey \& Lockman 1990). Spectra from the different instruments were fitted jointly using the same model parameters. The normalizations were not tied together, since their values are sensitive to assumptions about the spatial distribution of the X-ray emission and relative calibration of the detectors. Table 1 lists best fitting parameters for a few instrument combinations, together with the results of $\mathrm{ONC}$ for 


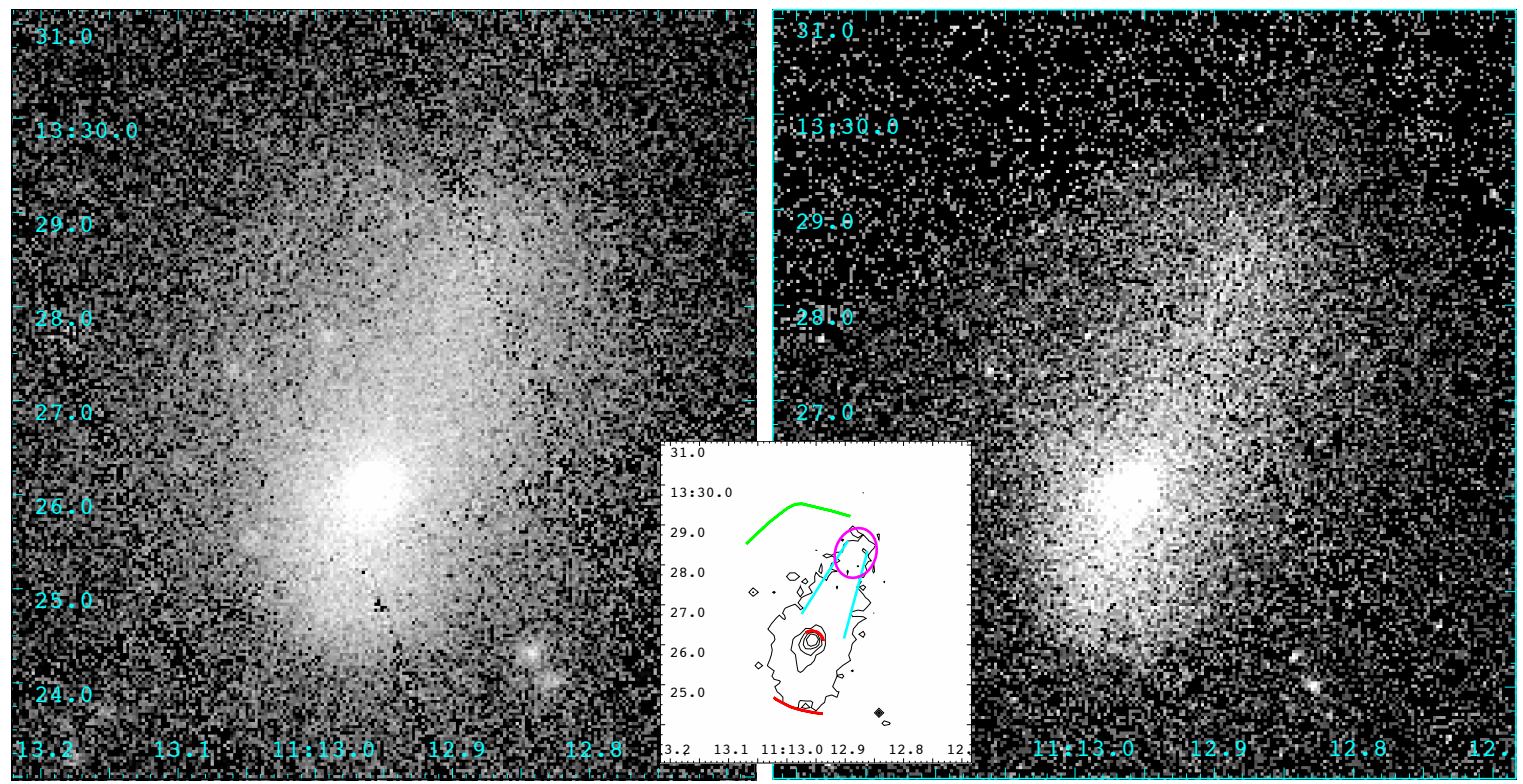

FIG. 1.- Exposure corrected, $0.5-7 \mathrm{keV}$ images from the EPIC data (Left) and from the ACIS data (Right). Both images have been binned to $2^{\prime \prime}$ per pixel. The substructures discussed in this paper are marked in color in the inset image, with X-ray contours in black: the two cold fronts (red), the offset core (magenta), the edge of the tail (green), and the ridge (cyan).

comparison. Uncertainties are $90 \%$ confidence limits. The net photon counts for the four detectors, given in the last column of the table, are similar. Thus, the total net photon count in the spectra used here is roughly four times greater than used by ONC. Nevalainen et al. (2010) found that temperatures determined from EPIC (PN and MOS) data are typically 10\% lower than temperatures for the same region determined from ACIS data when fitting in a broad band $(0.75-7 \mathrm{keV})$. This is roughly consistent with the temperature differences seen in our data (Table 1).

\subsection{Temperature and Abundance Maps}

ONClemployed the broad band method of Markevitch et al. (2000) to make a temperature map from the ACIS-S data. An improved map, made by applying the methods described in Randall et al. (2008) and Owers et al. (2011a) to the combined ACIS-I and ACIS-S data, is shown in the left panel of Figure 2 Briefly, the $0.5-7.0 \mathrm{keV}$, background subtracted images for ACIS-I and ACIS-S data were coadded and binned by a factor of 4 . At each pixel in the binned image, a spectrum was extracted from a circular region with radius set such that there are 1000 background-subtracted counts. The radius of the circles ranges from $6.7^{\prime \prime}$ at the center of cluster to $\sim 100^{\prime \prime}$ at the boundary of the map. The size of some extraction regions were marked with black circles in the left panel of Figure 2 Weighted response files were extracted from a more coarsely binned image with binning factor of 32 . The spectra were grouped to obtain at least 20 counts per channel. The data were fitted with an absorbed MEKAL model with the abundance fixed at $\mathrm{Z}=0.32$, i.e. the global value for the combined ACIS-S and ACIS-I fit in Table 1

A second temperature map was generated by fitting spectra binned into regions defined by a weighted Voronoi tesselation (WVT; Diehl \& Statler 2006) of the ACIS-I data. The target signal to noise ratio for the WVT binning was set to 25 , so that each spatial bin contains about 625 counts. Using the same regions, the spectra from all four data sets (ACIS-I, ACIS-S, MOS1, and MOS2) were extracted. Since the net $0.5-7 \mathrm{keV}$ count is comparable for all four data sets. Thus, the total net count for each spectral region lies in the range 1800 to 2800 , sufficient for robust temperature measurements. The signal to noise ratio for the WVT algorithm was chosen so that the smallest regions have a size comparable to the spatial resolution of XMM-Newton. Spectra were fitted using the absorbed MEKAL model as described above. Results of fitting spectra for the ACIS and MOS data separately and jointly were compared. Discrepancies between ACIS and MOS temperatures comparable to those discussed in Section 2.2 are seen, but the values generally agree within $1 \sigma$ statistical uncertainties. The temperature map in the right panel of Figure 2 shows results for the joint fit.

We present both temperature maps in Figure 2, since they have complementary properties. The maps in the left panel retains more spatial information, since it does not require the data to be binned on an artificial grid. However, the results in adjacent pixels are correlated over a distance that is not readily discerned from the maps. By contrast, the WVT map gives independent temperatures and abundances for welldefined regions, at the expense of binning together regions that may have disparate properties. The two temperature maps are broadly in agreement with one another. The primary cluster core (regions 2 of the bottom panels) is cool, with the cool gas extending southward. A stream of hotter gas crosses the bright ridge in the center of both maps. The subcluster core at the northern end of the ridge (region 1) is also marginally cooler than the surrounding regions.

The method used to make the high resolution temperature map of Figure 2 was used with coarser binning to obtain the temperature (left) and abundance (right) maps of Figure 3 In detail, the ACIS-I and ACIS-S data were binned by a factor of 8 and the spectrum at each pixel was extracted from a circular region containing at least 3000 background subtracted counts. The spectra were grouped to obtain at least one counts per channel. The minimal grouping is adopted to avoid diluting the $6.7 \mathrm{keV}$ iron line. Temperatures and abundances were fitted to an absorbed MEKAL model using the Cash statistics.

The abundance of the cool core is high. The inner and outer cold fronts are revealed in both the temperature and 

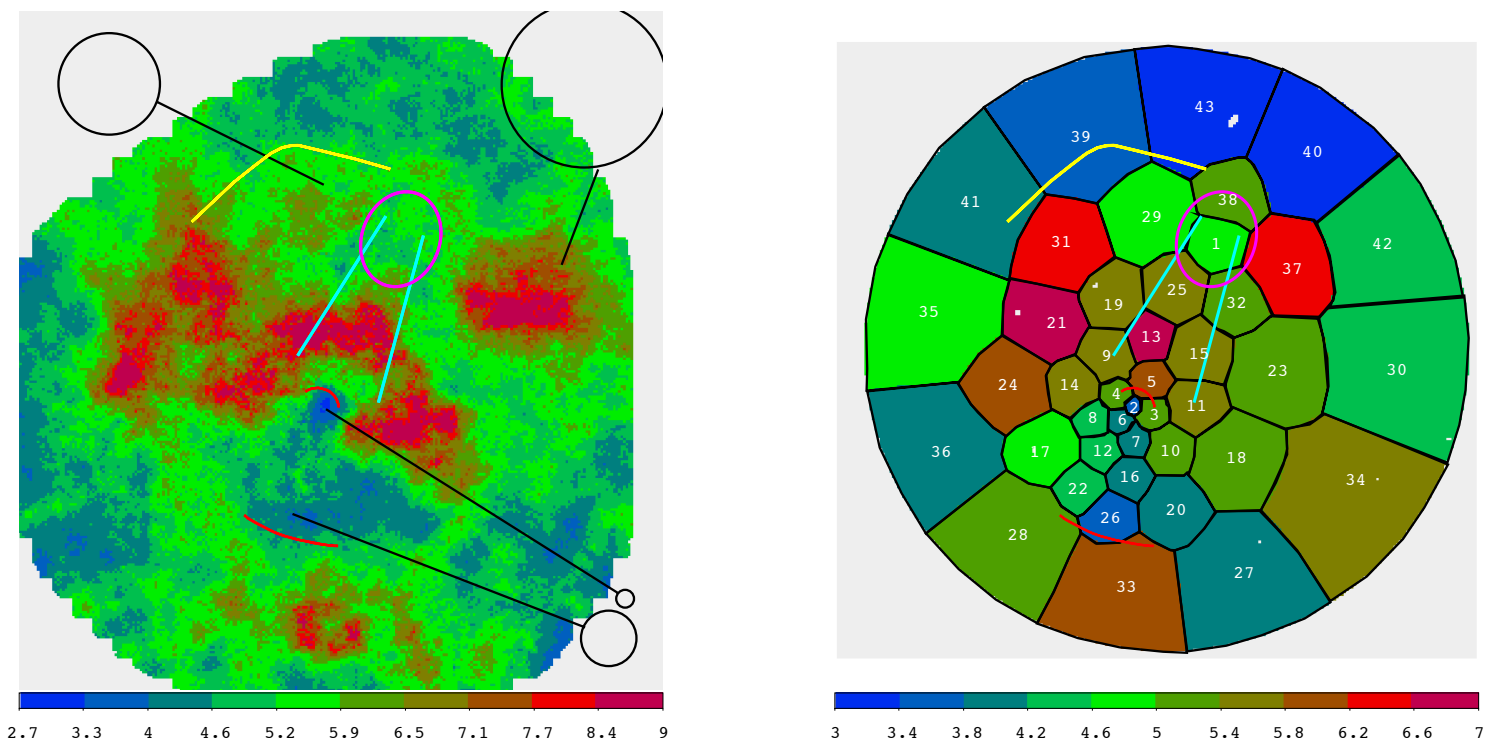

FIG. 2.- Left: High resolution temperature map. The temperature for each pixel is determined from the spectrum extracted within a circle containing 1000 background subtracted counts. The details are described in $\$ 2.3$ The median uncertainty (68\% confidence) ranges from $0.75 \mathrm{keV}$ at $k T \sim 4 \mathrm{keV}$ to $1.6 \mathrm{keV}$ at $T \sim 7 \mathrm{keV}$. The black circles mark the extraction regions at some sample pixels. Right: WVT temperature map from the combined ACIS+MOS data. Spatial bins were defined by applying the WVT binning algorithm to the ACIS-I data, with the signal to noise ratio set to 25 . Temperature uncertainties are about $0.5 \mathrm{keV}$ at $k T \sim 4 \mathrm{keV}$ and $0.7 \mathrm{keV}$ at $k T \sim 6 \mathrm{keV}$. Substructures of interest are marked on both panels, as in Figure 1
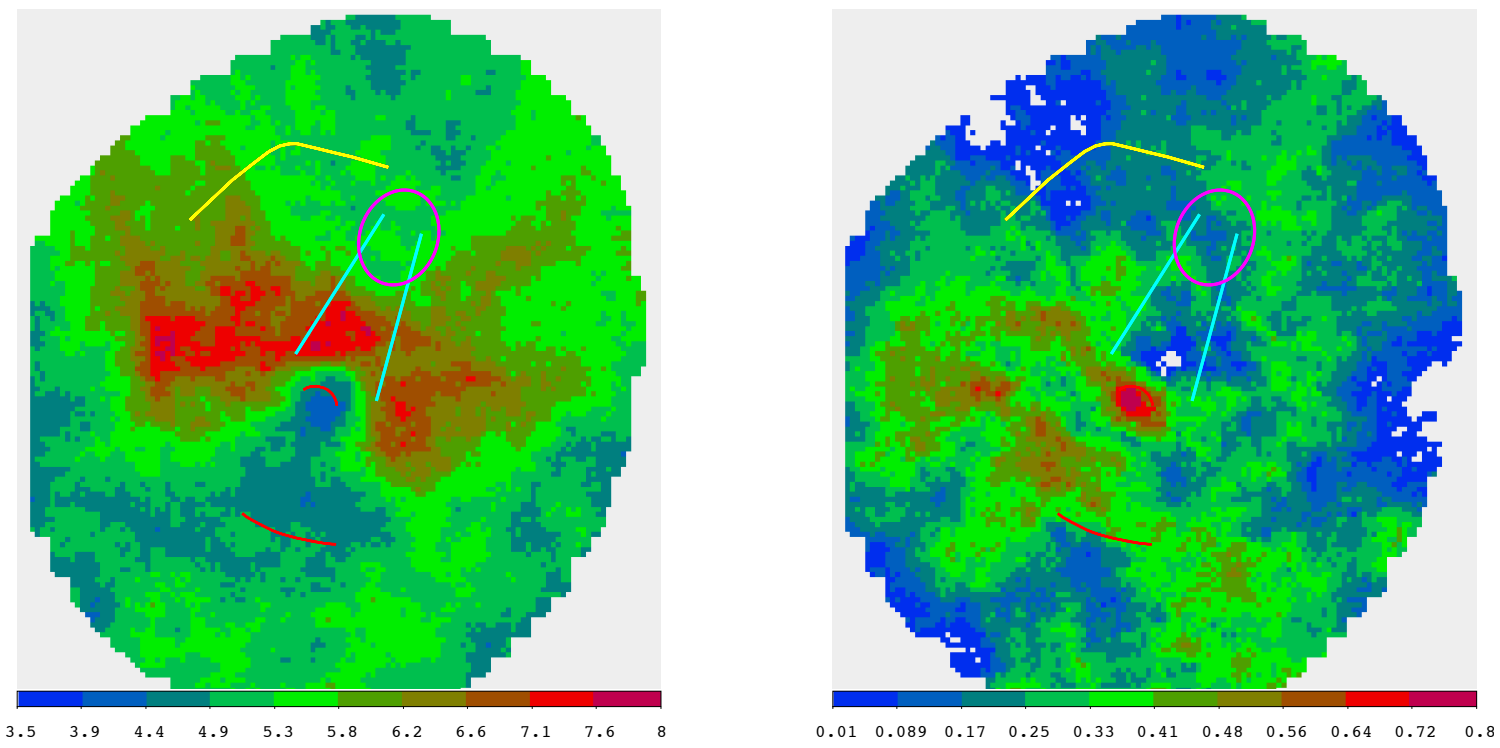

FIG. 3.- ACIS temperature (Left) and abundance (Right) maps. The temperature maps differs from the left panel of Figure 2 only by using spectra from regions containing 3000 background subtracted counts around each pixel. The same spectra were used to determine the abundances plotted in the right panel. The median temperature uncertainty ( $68 \%$ confidence) ranges from about $10 \%$ for $\mathrm{kT}<6 \mathrm{keV}$ to $20 \%$ for $\mathrm{kT}>6 \mathrm{keV}$. In the bright regions, the uncertainties on the abundance measurements are $\sim 0.1$ and rise to $\sim 0.2$ in the fainter regions. Substructures of interest are marked, as in Figure 1 and Figure 2 
abundance maps, with the abundance on the cooler side being higher than on the hotter side of both fronts. The ridge (between the cyan lines of Figure 3), as well as the offset core (the magenta ellipse), shows a marginally low abundance.

\subsection{Deprojected Gas Properties}

An "onion peeling" deprojection method was used to obtain radial temperature and density profiles for the gas of A1201 (David et al.2001; Nulsen et al.2005). Assuming that the gas distribution is spherical and that the gas densities and temperatures are constant in spherical shells, the algorithm uses spectral parameters fitted to the outer layers to calculate the spectra of outer shells projected onto inner annuli. Spectra were extracted for 7 annuli, centered on the cluster center and excluding the bright ridge (a sector in the range $\mathrm{PA}=50^{\circ}-$ $75^{\circ}$ ). The size of each annulus was adjusted to obtain about 3000 photons in each ACIS-I spectrum $(0.5-7.0 \mathrm{keV})$. The outer edge of the outermost annulus is about $700 \mathrm{kpc}$ from the cluster center. The fraction of the cluster emission in the outermost annulus arising from gas at larger radii is corrected using a beta model with $\beta=0.75$. The spectral model for each annulus is a sum of MEKAL models, all absorbed by the Galactic foreground column density. The parameters (temperature, abundance and norm) for all but one MEKAL component are determined by fits to the surrounding shells. Parameters for the remaining component are adjusted to fit the spectrum for the current annulus. Deprojection results are presented in Section 3.2

\section{MERGER RELATED STRUCTURES}

\subsection{Cold Fronts}

The new data were used to measure the surface brightness profiles and temperature jumps for the two cold fronts to confirm their properties with deeper data, following the procedure of ONC. Surface brightness profiles were constructed from the point-source-free ACIS $0.5-7 \mathrm{keV}$ event table, corrected for vignetting, quantum efficiency and exposure time. Background was determined from scaled blank sky data. For the northwest front, the profile was extracted in annuli centered on $\left(\right.$ R.A., DEC) $=\left(168.2268^{\circ}, 13.4344^{\circ}\right)$, for PA in the range $7^{\circ}-122^{\circ}$. Annuli for the southeastern front were centered at $($ R.A., DEC $)=\left(168.2197^{\circ}, 13.4509^{\circ}\right)$ with PA from $240^{\circ}-267^{\circ}$. The surface brightness profile is modeled, assuming that the electron density follows the broken power law

$$
n_{\mathrm{e}}(r)= \begin{cases}n_{\mathrm{e}, 1}\left(\frac{r}{R_{\mathrm{f}}}\right)^{-\alpha_{1}}, & r<R_{\mathrm{f}}, \\ n_{\mathrm{e}, 2}\left(\frac{r}{R_{\mathrm{f}}}\right)^{-\alpha_{2}}, & r>R_{\mathrm{f}},\end{cases}
$$

where $R_{\mathrm{f}}$ is the radius of the discontinuity and $n_{\mathrm{e}, 1}$ and $n_{\mathrm{e}, 2}$ are the inner and outer densities at $R_{f}$, respectively. Here, $r$ is the spherical radius. The small temperature dependence of the surface brightness is ignored. Surface brightness can then be determined by integrating $n_{\mathrm{e}}(r)^{2}$ along lines of sight. Model parameters were obtained by jointly fitting the ACISI and ACIS-S surface brightness profiles. The density jumps were found to be $n_{\mathrm{e}, 1} / n_{\mathrm{e}, 2}=1.74_{-0.30}^{+0.26}$ for the northwest discontinuity and $2.2_{-0.29}^{+0.27}$ for the southeast discontinuity ${ }^{8}$

Temperature jumps were determined by jointly fitting ACIS-I, ACIS-S and MOS spectra, using the method of ONC

\footnotetext{
${ }^{8}$ All uncertainties in this section are $90 \%$ confidence ranges.
}

Spectra for one region on each side of a front were extracted in the sectors defined above. For the northwest front, the region inside the front covered the range of radius $7^{\prime \prime}-17^{\prime \prime}$ and the region outside the front, $23.5^{\prime \prime}-50^{\prime \prime}$. For the southeast front, the radial ranges are $108^{\prime \prime}-175^{\prime \prime}$ (inside) and $190^{\prime \prime}-$ $280^{\prime \prime}$ (outside). The norm and temperature outside each front were fitted using an absorbed MEKAL model, with the metallicity fixed at the global value (0.29, see Table 1 ). Using these parameters and the results from fitting the broken power law model for the gas density, the norm for the gas outside the front projected onto the region inside the front was then calculated. This gives a two component model for the region inside the front, one component for the gas outside the front, with all parameters fixed, and a second component with free parameters for the gas inside the front. Fitting the free parameters to the spectrum for the region inside the front then determines the temperature of the gas there. The resulting temperatures are $3.15_{-0.38}^{+0.42} \mathrm{keV}$, inside, and $6.21_{-0.69}^{+1.5} \mathrm{keV}$, outside the northwest front. For the southeast front, they are $3.29_{-0.26}^{+0.34}$, inside, and $5.1_{-0.9}^{+1.7} \mathrm{keV}$, outside. Combining the density jumps determined from the surface brightness profile with the temperature jumps determined here, we find that the pressure jumps at the fronts are $0.86_{-0.22}^{+0.23}$ for the northwest front and $1.41_{-0.48}^{+0.50}$ for the southeast front. These do not differ significantly from 1, consistent with the results of $\mathrm{ONC}$ Thus, we confirm their conclusion that both fronts are cold fronts rather than shocks.

\subsection{Offset Core}

Using a KMM (Kayes mixture model; Ashman et al.|1994) analysis, ONC divided the 3-dimensional projected phase space of cluster members into a sum of Gaussians. Under this partitioning, $\mathrm{ONC}$ found a compact substructure at the location of the offset gas core, which is marked by the ellipse in Figure 1. This substructure has a velocity of $432 \mathrm{~km} \mathrm{~s}^{-1}$ relative to the cluster mean and a velocity dispersion of $166 \mathrm{~km} \mathrm{~s}^{-1}$. Although the X-ray emission from this region is not clearly distinct from the core of A1201, a pronounced ridge of X-ray emission extends from the core to the position of the substructure. We interpret this ridge as being composed of contributions from the large-scale cluster emission of A1201 and from gas associated with the offset core. In order to highlight emission from the offset core, a residual image, made by subtracting an elliptical beta mode $9^{9}$ fitted to the large-scale emission from the combined ACIS image, is shown in Figure 5. The cool core and the sector containing the southeast cold front were both masked out of the fit, since they are also not well-described by the beta model. The offset core stands out clearly at the end of the ridge.

The temperature and density of the offset core were estimated using spectra for the region marked by the magenta ellipse in Figures 1 and 5 . A local background was extracted from an annulus centered at the cluster center and containing the offset core, in the PA range $0^{\circ}-50^{\circ}$. Scaling this to the area of the ellipse to obtain a background spectrum and fitting an absorbed thermal model to the residual spectrum gives the excess emission measure of the offset core as $1.145_{-0.077}^{+0.085} \times 10^{66} \mathrm{~cm}^{-3}$. For a fixed emission measure in a fixed volume, the mean gas density is maximized when it is uniform. Assuming that the ellipse is the projection of a spheroid, with semi-major axis $a=110 \mathrm{kpc}$, semi-minor

\footnotetext{
${ }^{9}$ http://cxc.harvard.edu/sherpa4.2.2/ahelp/beta2d.html
} 

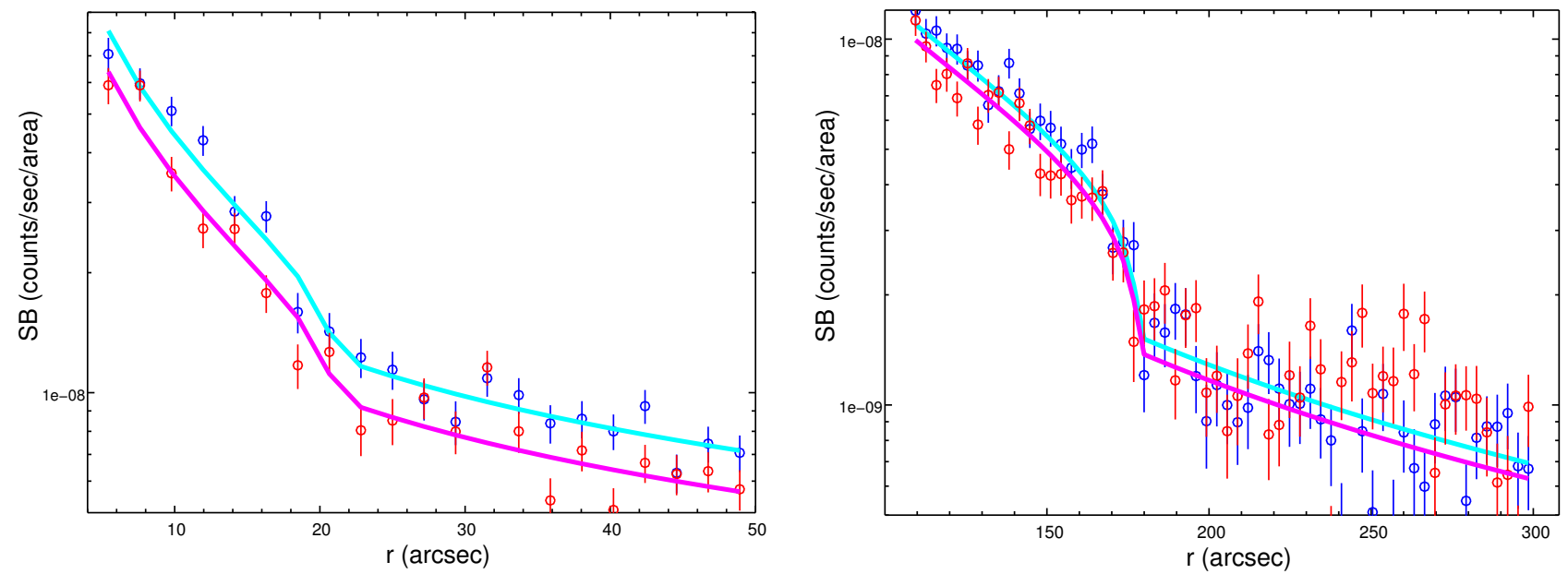

FIG. 4.- Surface brightness profiles for the inner (left panel) and outer (right panel) cold fronts. The red symbols and magenta lines shows data points and broken power law fits for the ACIS-S data. The blue symbols and cyan lines are for the ACIS-I data.

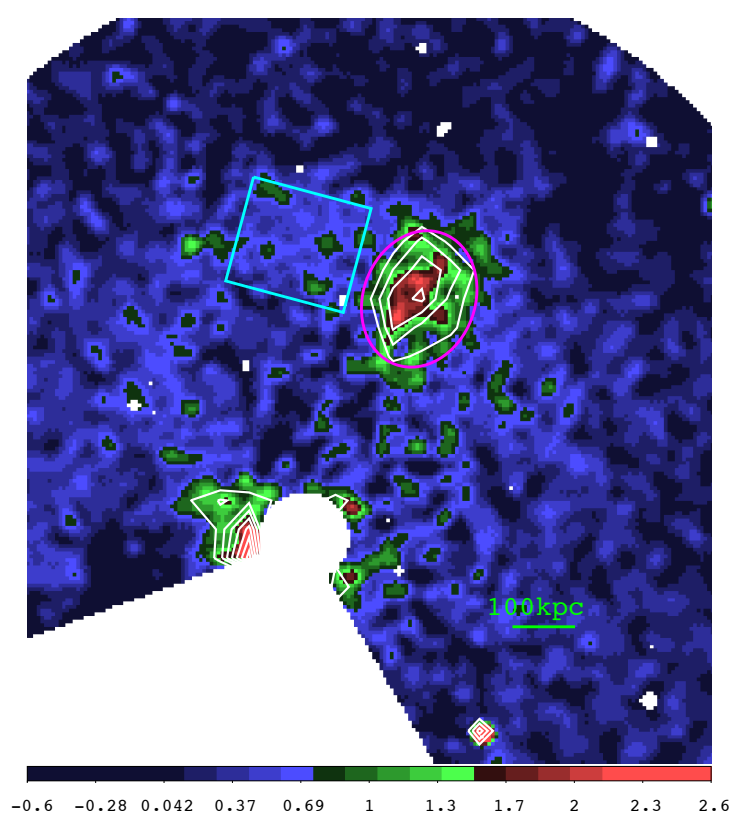

FIG. 5.- Residual image after subtraction of an elliptical beta model from the ACIS data. The image has been smoothed with a Gaussian kernel of radius $2^{\prime \prime}$. The magenta ellipse marks the offset core and the cyan square marks the region used to estimate the gas temperature and density of the tail.

axis $b=88 \mathrm{kpc}$, and its third semi-axis $c=\sqrt{a b}$, the electron density of the gas is $n=3.4 \pm 0.2 \times 10^{-3} \mathrm{~cm}^{-3}$. The uncertainty here is dominated by systematic errors, particularly in the volume of the emitting region. The errors show the range of densities as the third axis, $c$, is varied from the prolate $(c=b)$ to the oblate $(c=a)$ extremes. The corresponding gas mass of the offset core is $3.8 \pm 0.2 \times 10^{11} M_{\odot}$. The bright galaxy at (R.A., DEC) $=(168.2088,13.4750)$ is dynamically associated with the offset core, located close to its center, and has a stellar mass, estimated from SDSS photometry, of $\mathrm{M}_{\text {gal }}=3 \times 10^{11} M_{\odot}$, which is comparable to the gas mass. The second brightest galaxy associated with the offset core is one magnitude fainter. Only taking into account the stellar mass of the brightest galaxy and assuming that the ratio of the total mass to stellar mass is about 10 gives a conservative lower limit on the total mass of the offset core of $3 \times 10^{12} M_{\odot}$.
The temperature maps show that the offset core is only marginally cooler than surrounding regions (Figure 2). Since the offset core is brighter than adjacent regions at the same radius, it must be denser, as confirmed by the results above. In Figure 6, results for the temperature, density, pressure and entropy of the gas in the offset core are plotted in red together with the results of the deprojections. We note that the bright ridge was excluded from the spectra used for the deprojections, although this makes very little difference to the results. We also obtained a deprojected metallicity profile, which is consistent with the metallicity map in the right panel of Figure 3. The metallicity is highest in the cool core, $Z=0.86 \pm 0.2$ within $72 \mathrm{kpc}$ of the cluster center, and drops to $Z \sim 0.3-0.4$ at 140 to $690 \mathrm{kpc}$.

Although the gas temperature of the offset core is only marginally lower than that of the surrounding gas (top left panel of Figure 6, its entropy is significantly lower. This shows that the high density of the gas within the offset core is not simply the result of adiabatic compression of surrounding gas, since that would not alter the entropy. Thus, the gas in the offset core must originate in some other place. The simplest interpretation is that it is gas that fell in with the core. The abundance map in the right panel of Figure 3 shows marginal evidence of lower abundances near the offset core. While the significance of this feature is low, it is consistent with an origin for this gas outside the present cluster.

We can use the pressures to make a rough estimate of the direction of motion of the offset core. The thermal pressure of gas in the offset core is balanced by the external thermal pressure plus the ram pressure at the stagnation point. Using our estimates of the density and temperature from above gives an internal pressure for the core of $p_{\text {core }}=3 \times 10^{-11}$ dyne $\mathrm{cm}^{-2}$. The pressure of the external gas at the same radius is $p_{\text {ext }}$ is $10^{-11}$ dyne $\mathrm{cm}^{-2}$. Therefore, the ram pressure $p_{\text {rp }}$ is about $2 \times 10^{-11}$ dyne $\mathrm{cm}^{-2}$. Since the ram pressure $p_{\text {rp }}=\rho_{\text {ext }} v^{2}$, the velocity, $v$, of the offset core would need to be about $1000 \mathrm{~km} \mathrm{~s}^{-1}$.

Pressure arguments also constrain the location of the offset core along our line of sight. Both the thermal pressure and the ram pressure of the external gas depend on its density, which decreases with distance from the cluster center. If the distance of the offset core from the cluster center exceeds the projected separation, then the external gas pressure would be smaller, increasing the ram pressure required to confine the 

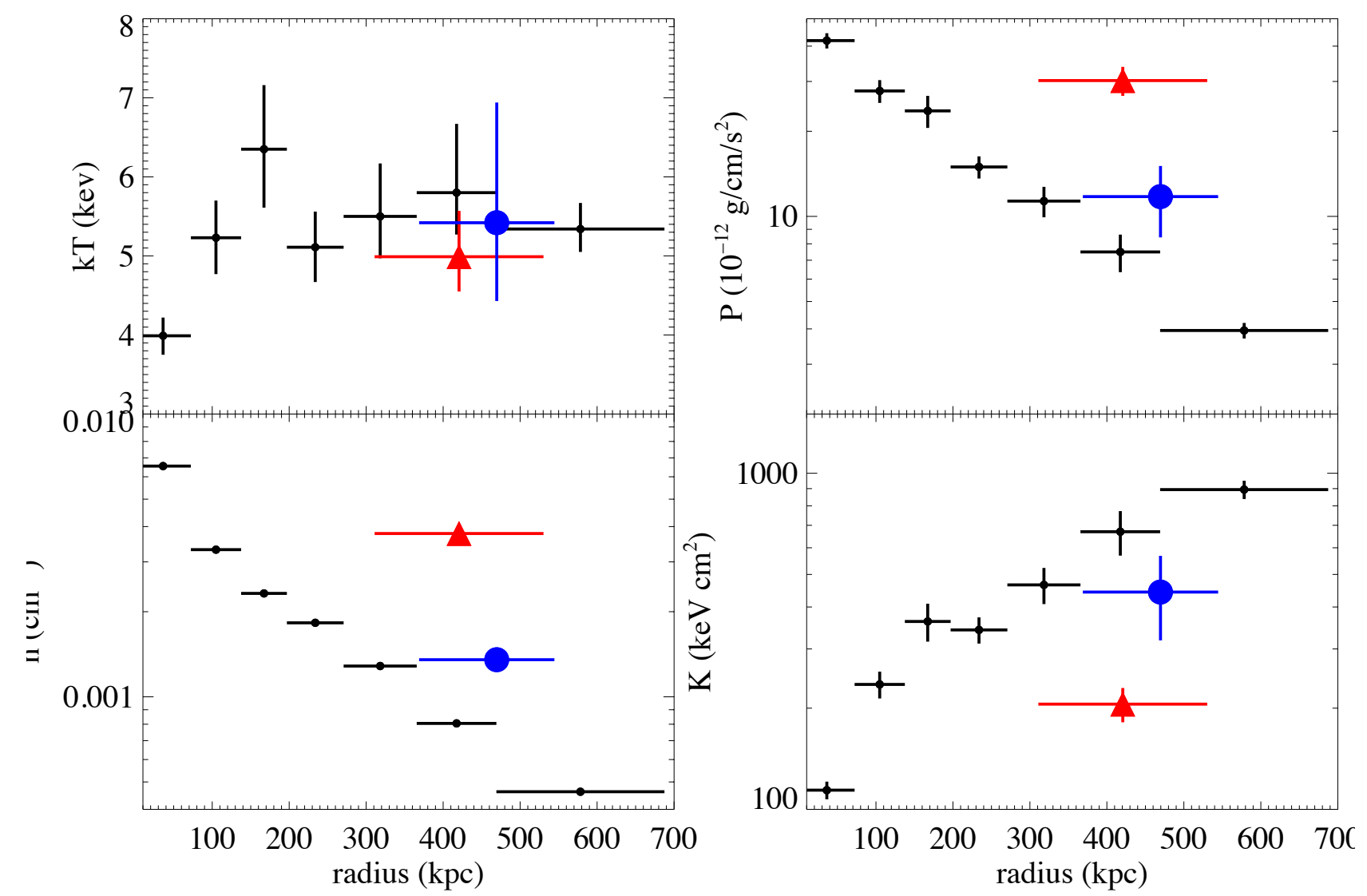

FIG. 6.- Deprojected temperature, density, pressure, and entropy profiles. Values are shown for the offset core (red triangle) and the tail (blue sphere) at the radii of the regions outlined in Figure 5 The uncertainties for some points are too small to be seen.

core. Since the gas density is also lower, a larger speed is then demanded to confine the gas in the offset core. However, in the absence of any evidence for a shock (ONC), we assume that the motion of the core is subsonic, i.e., its speed cannot exceed $\sim 1100 \mathrm{~km} \mathrm{~s}^{-1}$, which is not significantly higher than the velocity estimated above. Thus, the real distance of the offset core from the center of A1201 cannot be much greater than the projected separation of $\simeq 420 \mathrm{kpc}$. This requires the offset core to be located close to the plane of the sky.

\subsection{Tail of the Offset Core}

In the two panels of Figure 1, there is a region of brighter emission running to the northeast of the offset core that has a relatively sharp boundary to the north and a less well-defined edge to the east (marked by the green curve). We interpret the bright emission as a stream of gas being stripped from the offset core. Surface brightness profiles, obtained by the procedure of Section 3.1. for the northern edge of this feature (from box A in the upper panel of Figure 7) and its eastern edge (from box B) are shown in the lower panels of Figure 7 The surface brightness profile for box A shows a significant drop across the edge in all three data sets. This feature was not seen by ONC because of its limited coverage in the ACIS-S data, where the tail lies close to a chip gap. An abrupt drop in surface brightness can also be seen in the PN data (blue solid lines in the bottom panels of Figure 77 at the eastern edge of this feature, in the surface brightness profile for box B. The eastern edge is less significant in the noisier MOS and ACIS data, although they are reasonably consistent with the PN pro- file. Without the ACIS identification of the edge, the exact location of the eastern edge is not as securely constrained as the northern one. Profiles extracted from boxes C and D, on the opposite side of the bright ridge are shown for comparison. In them, the surface brightness fades smoothly away from offset core. Excess emission from the tail can also be seen in the residual map of Figure 5 .

In addition to the excess X-ray emission, the distribution of the galaxies associated with the offset core by the KMM algorithm is elongated in the direction of this tail (top panel of Figure 14 in (ONC). This is suggestive of a tidal tail of galaxies stripped from the disrupting core. However, the small number of galaxies in this structure, combined with the uncertainties in the KMM decomposition, make this hard to demonstrate with any certainty.

Although the tail does not stand out in the temperature or abundance maps of Figure 2 and 3 , both the temperatures and abundances are lower on the eastern side of the offset core, over the tail than they are to west. The absence of a significantly lower temperature in the tail may be simply because the stripped gas in the tail does not dominate the X-ray emission. To examine the properties of the tail more carefully, a spectrum was extracted from the tail region, defined as the rectangular region marked in Figure 5 Apart from scaling, the local background used is the same as that used to measure the spectrum of the offset core in Section 3.2 i.e., a sector from PA $0^{\circ}$ to $50^{\circ}$ of the annulus containing the offset core. X-ray emission in this region is dominated by the ICM of A1201, so that, after background subtraction, the spectrum 

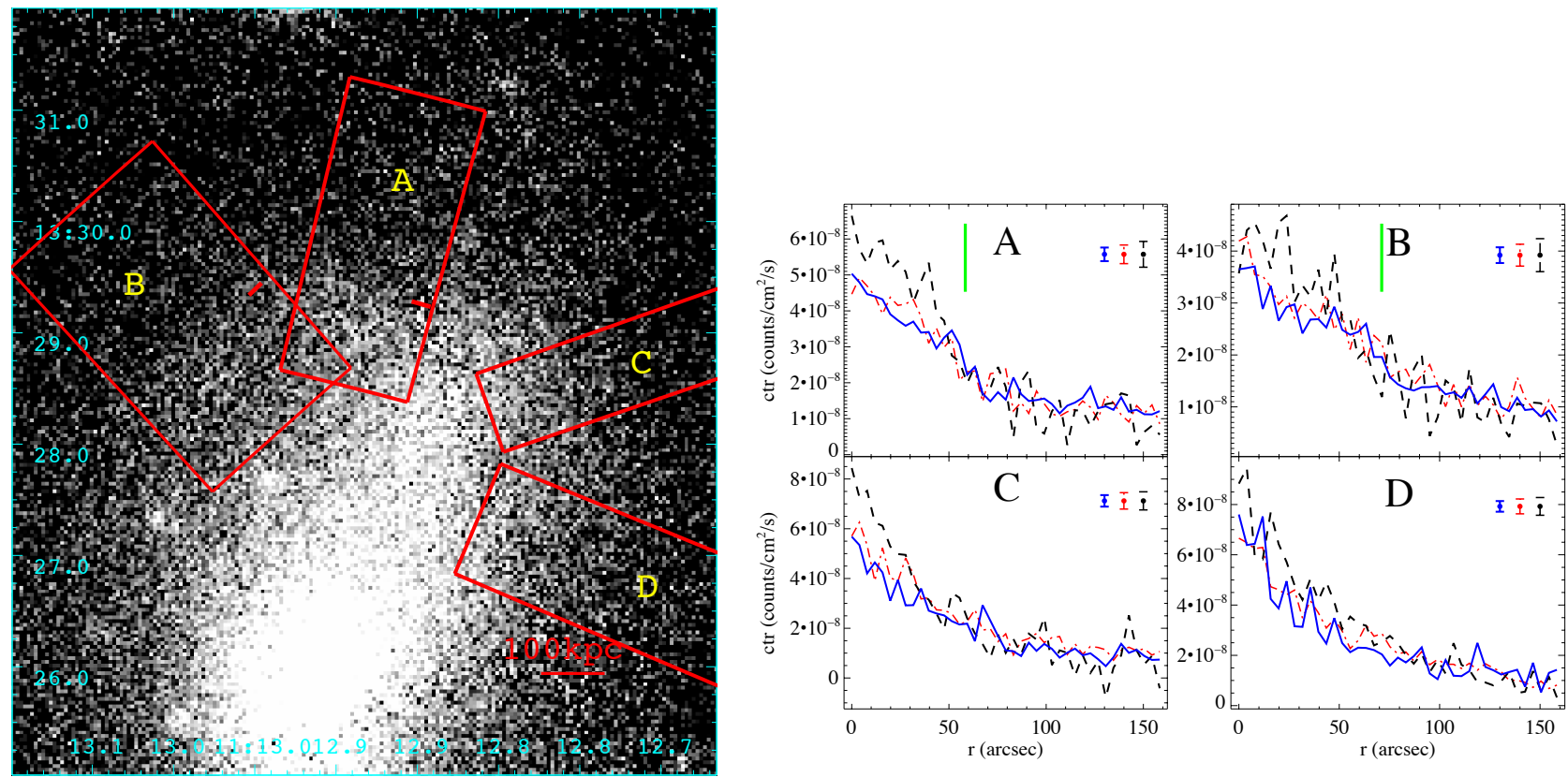

FIG. 7.- Top: EPIC image zoomed in on the northern tail. The four boxes show the regions used to extract surface brightness profiles shown in the lower panels: A, northern edge; B, eastern edge; C and D comparison regions. The locations of the edges are marked with ticks in regions A and B. Bottom: Surface brightness profiles for the four regions. Radius increases away from the cluster center in each panel, from zero at the inner edge of each box. Profiles for the merged ACIS data are plotted with black dashed lines, for the PN data with blue solid line, and for the MOS data with red dashed-dotted lines. The locations of the edges marked in green in the lower panels for boxes A and B correspond to the ticks in the boxes of the top panel. Typical statistical uncertainties are shown at the top right of each panel.

should be dominated by emission from the tail. These spectra for the ACIS and MOS data were fitted jointly using the absorbed thermal model of Section 2.2. The temperature of the tail was found to be $5.42_{-1.0}^{+1.5} \mathrm{keV}$. To estimate the density of the tail, we assume that the rectangular region from which the spectrum was extracted is the projection of a cylinder, with its height defined by the long side of the rectangle and its diameter by the short side. This gives an electron density for the tail of $1.31 \pm 0.1 \times 10^{-3} \mathrm{~cm}^{-3}$, where only the statistical uncertainty (90\% confidence level) is included. Although the systematic error is large, the density of gas in the tail is significantly lower than in the offset core (consistent with its lower surface brightness), but 1.7 times greater than the ICM density at the same radius (Figure 6).

The temperature and density determined here imply that the gas pressure of the tail is appreciably greater than that of its surroundings. In that case, the tail should expand at close to its internal sound speed, until it comes into pressure equilibrium with the surrounding gas. For the sound speed at $\sim 5 \mathrm{keV}$ and a tail width of $\simeq 100 \mathrm{kpc}$, this only requires $\simeq 0.1 \mathrm{Gyr}$. Taking the velocity of the offset core to be about $700 \mathrm{~km} / \mathrm{s}$ (see next section), the core would travel about $70 \mathrm{kpc}$ in this time, which should determine the length of the tail (projection effects can only reduce this). However, the extent of the tail is considerably more than $70 \mathrm{kpc}$ (see Figure 7). The most plausible cause of this discrepancy is that our estimates of the temperature and density of the tail do not accurately reflect its mean temperature and density. At typical cluster temperatures, X-ray brightness in detectors like ACIS and EPIC is quite insensitive to the gas temperature, being largely determined by its emission measure. Thus, our density estimate best represents the root-mean-square (RMS) density of the tail. Note that the excess brightness of the tail demands that its RMS density is higher than that of the surroundings. Stripped gas in the tail could be clumpy, making the RMS density larger than its mean density. Coupled to this, the temperature in the gas would almost certainly be non-uniform as well. Temperatures obtained by fitting single thermal models to emission from gas mixtures are not simply related to the mean temperature. Thus, our estimates of both of the temperature and density in the tail may not provide a good estimate of its mean pressure. It is more physically reasonable that most of the tail is close to local pressure equilibrium. Presumably, the gas is in the process of mixing into the ambient ICM. We note that to make the RMS density 1.7 times higher than the mean density, the distribution of gas densities in the tail would need to be broad. We also note that pressure estimate for the offset core may be affected similarly (in which case, the velocity estimate of section 3.2 would be high). However, because it stands out more clearly relative to the local cluster X-ray emission, our pressure estimate for the offset core should be less affected.

\section{DISCUSSION}

We begin by presenting our interpretation of the merger history of A1201 (Figure 8). On its initial core passage, the infalling subcluster was approaching us along a path to the southeast of the center of A1201 in projection. Much of the gas belonging to the subcluster would have been stripped during its first pericenter passage, although some gas has been retained by the subcluster core. The subcluster excited sloshing motions in the core of the primary cluster during its first core passage (Markevitch \& Vikhlinin 2007), giving rise to the cold fronts. The remnant subcluster core has now passed apocenter and it is close to its second pericenter passage, moving away from us. The orbit of the subcluster is roughly in a plane containing our line of sight $(-z$ direction in the figure) and the X-ray bright ridge. The evidence supporting this interpretation is outlined below.

\subsection{Orientation of the Orbit}




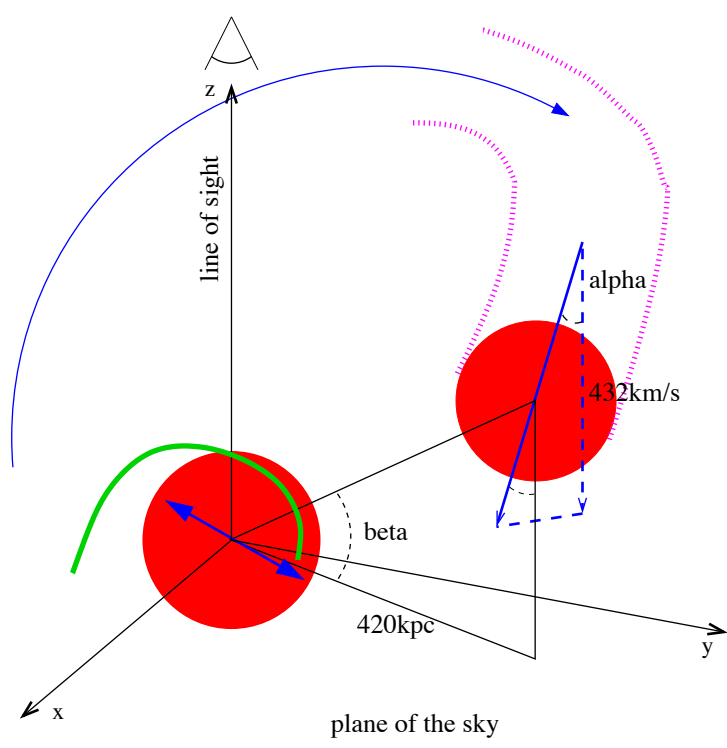

FIG. 8.- A simple merger model for A1201. Our line of sight is along the $z$ axis, from the $+z$ direction, while the $x-y$ plane is the plane of the sky. The sphere at the origin represents the core of the primary cluster and the other sphere represents the merging core. The blue solid vector from the offset core indicates its current direction of motion. The offset core is trailed by stripped gas within magenta dashed curves. The green curve represents the spiral front created by the sloshing motion excited in the merger. The blue arrows indicate the motion of the two cores.

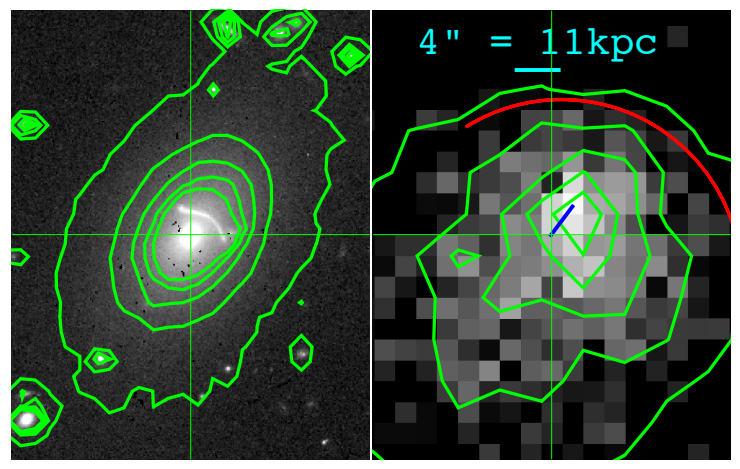

FIG. 9.- WFPC2 image of the BCG (left) and X-ray image of the cluster core (right). The WFPC2 image was made with the F606W filter (Edge et al. 2003. Surface brightness contours shown in each panel apply to the image in the panel. The red curve in the right panel marks the northwest cold front discussed in Secton 3.1 The cross marks the center of the BCG, which is offset from the peak of the X-ray emission by about $11 \mathrm{kpc}$ in projection. The major axis of the optical image is aligned with the direction of offset of the X-ray peak and the major axis of the X-ray emission.

Simulations of sloshing cold fronts show that contact discontinuities form along a spiral of enhanced X-ray emission that grows in the plane of the interloper orbit (Ascasibar \& Markevitch 2006, Poole et al. 2006). This feature is generally visible in $X$-ray images, unless the merger is viewed from close to the plane containing the spiral. Viewed from within its plane, the spiral appears as cold fronts on alternating sides of the cluster center. Since no spiral feature can be seen connecting the fronts in A1201, in either the X-ray image (Figure 1) or the temperature maps (Figure 2), it seems likely that we are viewing the spiral front from close to its plane, i.e. from close to the plane of the merging orbit.

In the X-ray images of A1201, a bright ridge lies along the axis joining the two sub-cluster cores. At least in part, this is composed of emission from the sloshing gas and the offset core. The two cold fronts are projections of the spiral feature, viewed from roughly within the plane of the spiral. In this orientation, enhanced X-ray emission from the whole of the spiral density feature (not just the cold fronts) is projected onto the nearly edge on plane of the orbit, contributing to the $\mathrm{X}$-ray emission from the bright ridge.

The simulations show that the spiral fronts created by sloshing wind from the outside inward in the prograde sense of the interloper orbit (e.g. Ascasibar \& Markevitch 2006; Poole et al. 2006). If the direction of the tail reveals the earlier path of the offset core, we expect that the core passed through its pericenter on the southeastern side of the center of the primary cluster, passing around it moving towards the northeast in projection. Thus, the spiral structure should wind from the inside out, counterclockwise, as plotted in Figure 8 and as projected onto the sky. The X-ray structure between the cold fronts is consistent with this scenario (Figure 3). We note that in simulations the outermost front generally appears on the opposite side of the cluster center to the pericenter passage that excited the sloshing (e.g. Roediger et al. 2011), whereas the outer cold front lies to the southeast in A1201. This might be an issue for our proposed merger orbit. However, in the simulation shown in Figure 7 of Ascasibar \& Markevitch (2006), the outer cold front changes sides at later times. This may well be a consequence of the second core passage. For our purpose, it only matters that these simulations demonstrate that the location of the outer front does not unambiguously determine the side of first core passage. This issue is further complicated by projection.

\subsection{Age of the Merger}

We can estimate the time elapsed since pericenter passage using the locations of the two cold fronts. First, if the sloshing motions that give rise to cold fronts may be regarded as a superposition of internal gravity waves excited by the merging subcluster (Churazov et al. 2003), we can estimate the time required for the cold fronts to get out of phase by $\pi$ radians as $\tau=\pi /\left(\omega_{\mathrm{BV} \text {,in }}-\omega_{\mathrm{BV} \text {,out }}\right)$, where $\omega_{\mathrm{BV}}$ is the Brunt-Väisälä frequency (Owers et al.|2011b; Simionescu et al.2011). This is given by

$$
\omega_{\mathrm{BV}}=\sqrt{\frac{g}{r} \frac{3}{5} \frac{d \ln \Sigma}{d \ln r}}=\omega_{\mathrm{K}} \sqrt{\frac{3}{5} \frac{d \ln \Sigma}{d \ln r}},
$$

where $r$ is the radius, $g=G M(r) / r^{2}$ is the acceleration due to gravity, $\Sigma$ is the entropy index and $\omega_{\mathrm{K}}=\sqrt{g / r}$ is the Kepler frequency. From observations (e.g Owers et al. 2011b), the factor under the square root on the right is close to unity, so that the approximation $\omega_{\mathrm{BV}} \simeq \omega_{\mathrm{K}}=V_{\text {circ }} / r$ is adequate for our purposes $\left(V_{\text {circ }}=\sqrt{g r}\right)$. To estimate $\omega_{\mathrm{K}}$, we measure radii from the cluster center, which is assumed to mark the potential minimum, and we assume that the mass distribution can be approximated as a singular isothermal sphere, so that the circular velocity is given by $V_{\text {circ }}=\sqrt{2} \sigma_{v}$, where $\sigma_{v}$ is the line of sight velocity dispersion. In A1201, the radii of the two cold fronts are $300 \mathrm{kpc}$ and $50 \mathrm{kpc}$, and $V_{\text {circ }}=1100 \mathrm{~km} \mathrm{~s}^{-1}$, giving $\tau \simeq 0.17$ Gyr. The Brunt-Väisälä frequency applies to purely transverse modes. For wavevectors with a radial component, the frequency is reduced by a factor of the sine of the inclination to the radial direction. Cold fronts are oriented almost radially, so this correction can be substantial. Owers et al. (2011b) and Roediger et al. (2011) found that the approximation in Eqn. 2 underestimates the time since pericenter passage by a factor of $3-4$. Correcting for this, we find that the first pericenter passage occurred $\sim 0.6 \mathrm{Gyr}$ ago. This 
age is smaller than expected if the merging core is close to its second pericenter passage. Note, however, that the 6:1 ratio of the radii of the two cold fronts in A1201 is substantially larger than the 4:1 ratio for the Virgo cluster fronts modeled by Roediger et al. (2011). Since the estimate above is largely determined by the location of the inner front, it may well be low.

The location of the outer front provides an alternative estimate of time elapsed since pericenter passage, assuming that the outer front was excited then. Roediger et al. (2011) found that cold fronts propagate outward with constant speed. For their Virgo cluster model, the outer front takes $1.5 \mathrm{Gyr}$ to reach a radius of $\sim 90 \mathrm{kpc}$. Given that $\mathrm{A} 1201$ is about twice as hot as the Virgo cluster, its characteristic speeds (sound speed and Kepler speed) should be a factor of $\simeq \sqrt{2}$ greater than for Virgo. The speed of the cold fronts scales with these, so that, scaling from the results of Roediger et al. (2011), the outer front in A1201 would require about 3.5 Gyr to reach its current radius of $300 \mathrm{kpc}$. This estimate leaves comfortably enough time for the merging subcluster to have reached its second pericenter passage. A more accurate estimate for the age of the merger requires a simulation better matched to A1201.

These arguments show that sufficient time has elapsed for the merging subcluster to have returned for its second core passage.

\subsection{Dynamics of the Offset Core}

As discussed in section 3.2, we estimate the speed of the offset core to be about $1000 \mathrm{~km} \mathrm{~s}^{-1}$, while the line of sight velocity of the core is $432 \mathrm{~km} \mathrm{~s}^{-1}$. To be consistent, these values require the core to be moving at an angle of about $65^{\circ}$ to our line of sight ( $\alpha$ in Figure 8). We note that this angle is distinct from the angle between the orbital plane of the offset core and the line of sight, as the direction of motion of the core within the orbital plane is not constrained (Figure 8). A relatively large value of $\alpha$ thus presents no conflict with the proposed model, which requires our line of sight to be nearly parallel to the orbital plane. The argument of $\$ 3.2$ assumes that the core is now subsonic. Note that if it was moving much faster than $1000 \mathrm{~km} \mathrm{~s}^{-1}$, its low line of sight velocity would require its motion to be even closer to the plane of the sky, increasing the likelihood that we could see the shock front it would create. This favors our assumption that its speed through the cluster is subsonic or, at least, only mildly supersonic.

As also argued in section 3.2 based on pressures, the distance of the offset core from the center of A1201 cannot be much greater than the projected separation of $\simeq 420 \mathrm{kpc}$. This requires the offset core to be located reasonably close to the plane of the sky. In other words, the angle $\beta$ in Figure 8 cannot be large.

The relatively high pressure in the offset core requires it to lie fairly close to the plane of the sky. Thus, we interpret the offset core as the remnant of a merging subcluster, close to its second core passage.

\subsection{Alignment of the Sub-Structures}

Edge et al. (2003) used the strong lensing arc projected onto the BCG (Figure 9) to calculate the mass distribution in the cluster center. They found the mass distribution to be elongated along the same axis as the BCG isophotes, but, interestingly, with a significantly greater ellipticity than the isophotes. Unfortunately, they found no lensed arcs further from the cluster center, nor enough galaxies for a weak-lensing analysis. Thus, their mass model is well-constrained only in the cluster core and the extent of the highly elliptical mass distribution is not known. At their time of publication, no high resolution X-ray image of A1201 had been obtained, leaving them unaware of the substructures discussed here.

The X-ray data combined with the optical data of ONC make a strong case for the presence of a remnant core at a distance of about $400 \mathrm{kpc}$ from the cluster center, in the direction of elongation of the mass distribution. This core is too far from the cluster center to contribute significantly to the mass distribution there. However, the disturbance in the dark matter and stars created by the merging core might well have elongated the mass distribution in this direction. It is remarkable that the major axis of the mass distribution, the offset between the BCG and the X-ray peak, and the cold fronts all lie along the same direction. Conventionally, alignments between cluster halos and their satellites are controlled by the orientation of large scale structures (e.g. Basilakos et al.2006). However, the large scale structure should not play an important role in A1201, as the location of the interloper changes rapidly at this stage of merging. Thus, the consistency of all alignments shown in A1201 suggests the possibility that the orientation of the BCG and the mass distribution in the cluster core are affected by the satellite directly. This would be surprising, since the mass in the cluster core should not be affected much by the tidal field of the interloper (cf. Faltenbacher et al. 2008). We speculate that alignment may be enhanced by sloshing, which would need to be verified by numerical simulations.

\section{SUMMARY}

We have analyzed the structure and dynamics of the merging cluster A1201 using the XMM-Newton and Chandra data. Structures associated with an infalling cluster, including cold fronts and an offset remnant core, were identified previously by ONC. In addition to these structures, the new data show enhanced emission east of the remnant gas core, with breaks in surface brightness along its boundary to the north and east. This is interpreted as a tail of gas stripped from the offset core.

Temperature and metallicity maps of A1201 made from the new data support the merger interpretation. High metallicity in the cool core which drops abruptly across the southern cold front is consistent with a sloshing cold front. There is a hint of reduced metallicity in the offset core and tail, as expected if these arise in an external system. Using the deprojected density, temperature, and entropy profiles of the cluster, the entropy of the gas in the offset core and the tail were found to be lower than in the gas at the same radius elsewhere in the cluster. This evidence is consistent with the offset core being the remnant gas of a merging satellite cluster and the tail being composed of gas stripped from it.

The observed properties of this system, including the placement of the cold fronts, the offset core and its tail, together with our estimate for the velocity of the offset core are consistent with a simple merger model for A1201 that is sketched in Figure 8. In this model, the offset core passed pericenter to the southeast of the primary cluster core and it is now on its second pericenter passage, moving with a transonic velocity of $\sim 1000 \mathrm{~km} \mathrm{~s}^{-1}$. The compact, marginally low temperature, structure of the offset core indicates that this gas belongs to the interloper, having survived the first core passage and the gas is being stripped to form the tail behind it. The gas in the primary core was perturbed by the infalling core, causing the sloshing that gave rise to the two visible cold fronts. 
The disposition of the cold fronts requires that the merger is viewed from close to the plane containing the orbit of the interloper. Moreover, the remarkable alignment between the major axis of the mass distribution in the cluster core, the offset between the BCG and the X-ray peak, the cold fronts and larger scale structure suggests that they have all been affected by the merger disturbing the core of the primary cluster.

This work was partly supported by NASA grants NAS803060 and NNX08AD68G. CJM and BRM are supported by
Chandra Large Project Grant: G09-0140X. BRM acknowledge generous support from the Natural Sciences and Engineering Research Council of Canada. MSO and WJC acknowledge the financial support of the Australian Research Council. We have made use of data obtained under the Chandra HRC GTO program and software provided by the Chandra X-ray Center (CXC) in the application packages CIAO, ChIPS, and Sherpa. STSDAS is a product of the Space Telescope Science Institute, which is operated by AURA for NASA.

\section{REFERENCES}

Ascasibar, Y., \& Markevitch, M. 2006, ApJ, 650, 102

Ashman, K. M., Bird, C. M., \& Zepf, S. E. 1994, AJ, 108, 2348

Basilakos, S., Plionis, M., Yepes, G., Gottlöber, S., \& Turchaninov, V. 2006, MNRAS, 365, 539

Böhringer, H., et al. 2000, ApJS, 129, 435

Carter, J. A., \& Read, A. M. 2007, A\&A, 464, 1155

Churazov, E., Forman, W., Jones, C., \& Böhringer, H. 2003, ApJ, 590, 225

David, L. P., Nulsen, P. E. J., McNamara, B. R., Forman, W., Jones, C., Ponman, T., Robertson, B., \& Wise, M. 2001, ApJ, 557, 546

Dickey, J. M., \& Lockman, F. J. 1990, ARAA, 28, 215

Diehl, S., \& Statler, T. S. 2006, MNRAS, 368, 497

Ebeling, H., Edge, A. C., Böhringer, H., Allen, S. W., Crawford, C. S., Fabian, A. C., Voges, W., \& Huchra, J. P. 1998, MNRAS, 301, 881

Edge, A. C., Smith, G. P., Sand, D. J., Treu, T., Ebeling, H., Allen, S. W., \& van Dokkum, P. G. 2003, ApJ, 599, L69

Faltenbacher, A., Jing, Y. P., Li, C., Mao, S., Mo, H. J., Pasquali, A., \& van den Bosch, F. C. 2008, ApJ, 675, 146

Fruscione, A., et al. 2006, CIAO: Chandra's data analysis system

Johnson, R. E., Markevitch, M., Wegner, G. A., Jones, C., \& Forman, W. R. 2010, ApJ, 710, 1776

Johnson, R. E., ZuHone, J. A., Jones, C., Forman, W., \& Markevitch, M. 2011, Sloshing Gas in the Core of the Most Luminous Galaxy Cluster RXJ1347.5-1145, 17 pages, 5 figures; Corrected author and affiliation list Markevitch, M. 1998, ApJ, 504, 27

Markevitch, M., Gonzalez, A. H., David, L., Vikhlinin, A., Murray, S., Forman, W., Jones, C., \& Tucker, W. 2002, ApJL, 567, L27

Markevitch, M., \& Vikhlinin, A. 2007, Phys. Rep., 443, 1

Markevitch, M., Vikhlinin, A., \& Mazzotta, P. 2001, ApJ, 562, L153
Markevitch, M., et al. 2000, ApJ, 541, 542

Mewe, R., Lemen, J. R., \& van den Oord, G. H. J. 1986, A\&As, 65, 511

Nevalainen, J., David, L., \& Guainazzi, M. 2010, A\&A, 523, 22

Nulsen, P. E. J., McNamara, B. R., Wise, M. W., \& David, L. P. 2005, ApJ, 628,629

Owers, M. S., Randall, S. W., Nulse, P. E. J., Couch, W. J., David, L. P., \& Kempner, J. C. 2011, ApJ, 728, 27

Owers, M. S., Nulsen, P. E. J., \& Couch, W. J. 2011, ApJ, 741, 122

Owers, M. S., Nulsen, P. E. J., Couch, W. J., \& Markevitch, M. 2009a, ApJ, 704, 1349

Owers, M. S., Nulsen, P. E. J., Couch, W. J., Markevitch, M., \& Poole, G. B. 2009b, ApJ, 692, 702, (ONC)

Poole, G. B., Babul, A., McCarthy, I. G., Sanderson, A. J. R., \& Fardal, M. A. 2008, MNRAS, 391, 1163

Poole, G. B., Fardal, M. A., Babul, A., McCarthy, I. G., Quinn, T., \& Wadsley, J. 2006, MNRAS, 373, 881

Randall, S., Nulsen, P., Forman, W. R., Kones, C., Machacek, M., Murray, S. S., \& Maughan, B. 2008, ApJ, 473, 651

Roediger, E., Brüggen, M., Simionescu, A., Böhringer, H., Churazov, E., \& Forman, W. R. 2011, MNRAS, 413, 2057

Sarazin, C. L. 2002, in Merging Processes in Galaxy Clusters, Vol. 272, $1-38$

Simionescu, A., et al. 2011, Sci, 331, 1576

Springel, V., Frenk, C. S., \& White, S. D. M. 2006, Nature, 440, 1137

Struble, M. F., \& Rood, H. J. 1999, ApJS, 125, 35

Tittley, E. R., \& Henriksen, M. 2005, ApJ, 618, 227

Vikhlinin, A., Markevitch, M., \& Murray, S. S. 2001, ApJ, 551, 160

ZuHone, J. A., Markevitch, M., \& Johnson, R. E. 2010, ApJ, 717, 908 